INVESTIGACIÓN

https://doi.org/10.15198/seeci.2019.48.109-123

Recibido: 21/04/2018 --- Aceptado: 22/01/2019 --- Publicado: 15/03/2019

\title{
EVALUACIÓN DE LOS PODCASTS DE NOTICIAS: UN ACERCAMIENTO A EMISORAS DE LA CIUDAD DE PUEBLA
}

\section{EVALUATION OF NEWS PODCASTS: AN APPROACH TO BROADCASTERS OF PUEBLA CITY}

Paola Eunice Rivera Salas ${ }^{1}$ : Facultad de Ciencias de la Comunicación, Benemérita Universidad Autónoma de Puebla. Puebla, México. paola.rivera@correo.buap.mx

Octavio Ricardo Montoya Márquez: Facultad de Ciencias de la Comunicación, Benemérita Universidad Autónoma de Puebla. Puebla, México.

octaviomontoya95@gmail.com

\section{RESUMEN}

El podcast es una de las diversas herramientas colaborativas que se implementan en el Periodismo Digital, siendo un formato de fácil distribución y que puede proporcionar un espacio dinámico y creativo para el profesional de la comunicación social. Esta investigación pretende evaluar los podcasts que se publican en sitios de noticias de la ciudad de Puebla, México. Las variables que se consideraron fueron: función, producción y difusión del podcast. La investigación fue de tipo cualitativa, descriptiva, de corte transversal y no experimental. La información se recolectó a partir de una guía de observación. Las categorías utilizadas para la evaluación de las grabaciones fueron: producción, función periodística y difusión. Los resultados arrojaron que los podcasts son, en su mayoría, grabaciones íntegras o parciales de los programas en vivo. Su función es primordialmente informativa, y no se aplica como un canal para el periodismo de investigación. Además, se identificó que las emisoras no dan seguimiento a las ligas y a la interacción que los propios podcasts generan.

PALABRAS CLAVE: podcast- podcasting- periodismo digital- portales de noticias ciberperiodismo - producción - difusión.

\section{ABSTRACT}

The podcast is one of the diverse collaborative tools that are implemented in Digital Journalism, being an easily distributed format that can provide a dynamic and

${ }^{1}$ Paola Eunice Rivera Salas: Doctora en Educación (UVHM). Profesora - Investigadora de la Facultad de Ciencias de la Comunicación de la BUAP. Miembro del Cuerpo Académico "Comunicación y Sociedad".

paola.rivera@correo.buap.mx 
creative space for the social communication professional. This piece of research aims to evaluate the podcasts that are published on news sites in the city of Puebla, Mexico. The variables that were considered were: function, production and dissemination of the podcast. Research was qualitative, descriptive, cross-sectional and non-experimental. The information was collected from an observation guide. The categories used for the evaluation of the recordings were: production, journalistic function and dissemination. The results showed that podcasts are, mostly, full or partial recordings of live programs. Their function is primarily informative and is not applied as a channel for investigative journalism. In addition, it was identified that the stations do not track the leagues and the interaction that podcasts themselves generate.

KEY WORDS: podcast - podcasting - digital journalism - news portals cyberjournalism - production - diffusion.

\section{AVALIAÇÃO DOS PODCASTS DE NOTÍCIAS: UMA APROXIMAÇÃO À EMISSORAS DA CIDADE DE PUEBLA}

\section{RESUME}

O podcast é uma das diversas ferramentas colaborativas que se implementam no Jornalismo Digital, sendo um formato de fácil distribuição e que pode proporcionar um espaço dinâmico e criativo para o profissional da comunicação social. Esta investigação pretende avaliar os podcasts que se publicam em lugares de notícias da cidade de Puebla, México. As variáveis que se consideraram foram: função, produção e difusão do podcast. A investigação quantitativa, descritiva, de corte transversal e não experimental. A informação se correlatou a partir de uma guia de observação. As categorias utilizadas para a avaliação das gravações foram: produção, função jornalística e difusão. Os resultados mostraram que os podcasts são, em sua maioria, gravações integras ou parciais dos programas ao vivo. Sua função é primordialmente informativa, e não se aplica como um canal para o jornalismo de investigação. Ademais, se identificou que as emissoras não dão seguimento às ligas e à interação que os próprios podcasts geram.

PALAVRAS CHAVE: podcasts - podcasting - jornalismo digital - portais de notícias - ciberjornalismo - produção - difusão.

\section{Cómo citar el artículo:}

Rivera Salas, P. E., y Montoya Márquez, O. R. (2019). Evaluación de los podcasts de noticias: un acercamiento a emisoras de la ciudad de Puebla. [Evaluation of news podcasts: an approach to broadcasters of Puebla city]. Revista de Comunicación de la SEECI, 48, 109-123. doi: http://doi.org/10.15198/seeci.2019.48.109-123 Recuperado de http://www.seeci.net/revista/index.php/seeci/article/view/543 
Rivera Salas, P. E., y Montoya Márquez, O. R. Evaluación de los podcasts de noticias: un acercamiento a emisoras de la ciudad de Puebla

\section{INTRODUCCIÓN}

Con el despegue de las TIC, muchas empresas de diversos giros deciden explorar la vía de Internet con un alto nivel de incertidumbre, ya que no saben exactamente qué está sucediendo en el espacio virtual. Sin embargo, tienen la certeza de que, ante los cambios, es mejor estar ahí y aprender este nuevo entorno, que permitir que alguien ocupe su nicho de mercado.

Sin duda, empresas tales como las radiodifusoras han transitado por este mismo dilema. El Internet es un nuevo canal que cada vez se fortalece como un espacio de millones de consumidores/usuarios. Dicho espacio, a raíz del surgimiento de la Web 2.0, se ha venido complejizando. Así, cada día incrementa su oferta respecto a un gran número de formatos muy útiles para dar a conocer información de todo tipo.

En palabras de Díaz Noci y Meso (1999: 21, citado en Jarque-Muñoz \& AlmironRoig, 2008), el periodismo se traslada al formato multimedia debido a: 1) su incapacidad de aumentar e incluso mantener el número de lectores; 2) el aumento de la información y servicios que no son compatibles con el periódico impreso; 3 ) la diversificación de la actividad empresarial en medios de comunicación; 4) la escasez de insumos como el papel, en contraste con el aumento del impacto ambiental que este medio produce; y 5 ) los avances técnicos, como el constante desarrollo de los ordenadores.

Desde hace más de una década, autores como Díaz Noci \& Salaverría (2003), o Izquierdo-Labella (2012), coinciden en que el periodismo está en una etapa digital, en que las noticias ya no sólo se muestran como textos redactados. Las líneas informativas ahora vienen acompañadas con contenidos digitales diseñados para maximizar la interactividad y la hipertextualidad.

Es así como este documento se centra en una de las herramientas que se han aplicado como forma alternativa de presentar la información. Para ello, la investigación presenta una breve evaluación sobre los podcasts que algunas emisoras han publicado. Asimismo, se señalan las áreas de oportunidad que tienen dichas transmisoras, a fin de utilizar esta herramienta de manera más eficiente.

\subsection{Consideraciones teóricas}

\subsubsection{El podcast y los portales de noticias}

A todo el inmenso universo de la actual innovación tecnológica se suma de manera importante una herramienta de amplio poder comunicativo: el podcast. ¿Qué es el podcast? Un archivo digital de contenido exclusivamente auditivo, que se genera mediante un registro de voz con opción a otros elementos auditivos -ruido ambiental, música, efectos-. Para que un archivo de este tipo se pueda considerar meramente un podcast, es indispensable que sea accesible para todo usuario con conexión a Internet: en un blog, red social, página web, portal de noticias, etcétera. 
En suma, un podcast es un archivo de audio -o un conjunto estrechamente relacionado de ellos-, que se generan con diversos propósitos, formato, temática, estilo, y que puede ser "subido" a la Red para su permanente resguardo y escucha. En este sentido, el podcasting es la forma en que se distribuyen este tipo de archivos a toda la Web -echando mano de la sindicación- de modo que esté disponible en todo tipo de interfases (Laaser, Jaskilioff, Rodríguez y Lía, 2010).

El podcast es una herramienta que se ha visto potenciada por la interacción y la accesibilidad que proporciona el Internet; en gran parte gracias a la aparición de la Web 2.0. Se ha vuelto cada vez más utilizado, ya que casi cualquier usuario con un simple dispositivo de registro de sonido, y una aplicación digital de grabación y edición, puede crear su propio podcast (Solano y Sánchez, 2010).

El podcast, como herramienta de almacenamiento y difusión de información, tiene ciertas ventajas respecto a otros formatos del medio digital. Entre ellas, podemos mencionar su corta duración -que facilita su escucha completa-; su bajo consumo de energía y de datos -para los dispositivos en los que es escuchado-; no se requiere que el usuario vea permanentemente la pantalla de su dispositivo; la posibilidad de "seguir" o suscribirse a canales específicos; relativamente baja inversión de recursos y de tiempo en su elaboración; buena oportunidad para la mercadotecnia, pues no se pueden saltar los anuncios, además de que sirve para segmentar públicos; involucra una sensación de personalización; al no ser radio convencional, está impregnado de un aura de innovación (Bullido, 2015).

\subsubsection{La función informativa del podcast de noticias}

La noticia hoy en día sigue siendo la pieza clave y la razón de ser de los medios. No obstante, el punto medular para los editores es aprender a discernir cuál información, entre toda la que se genera y fluye cotidianamente, es realmente una noticia. Además, y como en tiempos pasados, es importante evitar que las personas o empresas utilicen el género noticioso como una perniciosa forma de autopromocionarse: ahora mediante la potencial enorme difusión alcanzable gracias a las herramientas a las que se tiene acceso en la Era Digital.

En los cibermedios las noticias han alcanzado mayor protagonismo que en los medios impresos. Por una parte, se ha logrado propagar verdaderamente información de "última hora". Por otra parte, con el ciberperiodismo el entorno se ha llenado de tintes interpretativos, pues el cibernauta, a partir de encabezados atractivos y simples, se siente atraído por el contenido y las implicaciones que tiene cada noticia (Salaverría, 2005). De este modo, se dan las condiciones hipertextuales facilitadoras para que cada ususario abunde en el tema de su interés.

Por otro lado, Izquierdo-Labella (2012) afirma que los géneros periodísticos que más tendrán peso en el formato digital serán la entrevista y el reportaje, puesto que lo multimedia permite exponer distintas voces, sonidos, textos e imágenes, que resultan a la postre atractivos a las audiencias. Este autor también subraya el 
Rivera Salas, P. E., y Montoya Márquez, O. R. Evaluación de los podcasts de noticias: un acercamiento a emisoras de la ciudad de Puebla

surgimiento del post o el tweet como nuevas géneros que prevalecerán durante un buen tiempo en la red.

Como ya se dijo antes, con el surgimiento de las herramientas Web 2.0 se facilita en muchos ámbitos comunicativos la posibilidad de obtener los resultados deseados por las diversas organizaciones, empresas e instituciones que se insertan en lo digital. Las cuales pueden tener objetivos tan diversos como transmitir conocimientos, obtener información y contenidos pertinentes desde prácticamente cualquier región del mundo, crear redes de especialistas e interesados en tópicos en común, producir de manera colaborativa; entre otros (Laaser, Jaskilioff, Rodríguez y Lía, 2010).

Por otra parte, y ya en el ámbito noticioso, Briggs (2007) asegura que a menudo los profesionales del periodismo llevan a cabo reportajes o entrevistas que en la versión impresa no logran transmitir la emoción que el entrevistado expresó en el momento del proceso de obtención de información. $Y$ es precisamente por lo anterior que asevera que gran parte de los trabajos de un medio periodístico digital saldrían beneficiados si cuentan con un podcast anexo -al cual se podría acceder con un hipervínculo, por ejemplo-.

\subsubsection{La producción del podcast}

A partir de la década pasada -e incluso antes-, diversos periódicos han estado produciendo podcasts. En Estados Unidos de América, por ejemplo, se tiene a The Naples Daily News, The New York Times, The Washington Post y The San Francisco Chronicle (Briggs, 2007). En la República Mexicana destaca el Grupo Televisa, empresa que ha generado grabaciones de forma continua para su noticiero matutino. Dichos podcasts abordan los titulares del día, noticias de todos los géneros, y hasta publicidad.

Hay que decir que en muchos medios digitales la mayoría de los podcasts nacen como webcasts. emisiones de radio en tiempo real. Posteriormente, estas grabaciones son almacenadas e indexadas en sitios específicos; y de este modo se convierten propiamente en podcasts (Solís, 2010).

Se ha dicho líneas arriba que el Podcast es una gran herramienta hipermediática de nuestros días. Uno de sus grandes atributos que le dan esa distinción es la facilidad relativa en su elaboración. Sin embargo, hay que hacer una pausa necesaria en este punto: para que un podcast pueda considerarse profesional, hay que cumplir con ciertos lineamientos técnicos, de producción y de contenido. Se necesita contar con un guion bien pensado, que oriente a los objetivos del producto. Después, es menester tener cuidado con los aspectos técnicos -grabación clara y de calidad, ruido ambiental, fondeo, música, efectos, dicción del locutor o locutores, entre otros-. Por último -y más importante-, se necesita que lo expresado sea interesante, fácil de entender, y que motive la escucha del público al que está dirigido (Laaser, Jaskilioff, Rodríguez y Lía, 2010). 
Rivera Salas, P. E., y Montoya Márquez, O. R. Evaluación de los podcasts de noticias: un acercamiento a emisoras de la ciudad de Puebla

Un aspecto más en el que las emisiones de radio tradicional se asemejan al podcast, es que éste último puede del mismo modo tener una gran cantidad de formatos, estilos y contenidos: de información local, nacional e internacional; de discusión, cultural, de difusión, de entrevista, un editorial, etcétera. En suma, es una herramienta que posibilita el pleno uso de la creatividad de quien lo elabora (Quintanal, 2012), pues se pueden usar o no efectos, música, sólo voz, sonido ambiente, o todas las combinaciones que se deseen, en el orden y frecuencia que se quiera. Por supuesto, siempre en aras de conseguir los objetivos planteados de antemano.

Un recordatorio pertinente respecto a la producción de podcasts, se da en relación a que no es muy provechoso ser ni serios ni formales en demasía. Es decir, no se debe confundir los contenidos trascendentes y rigurosos, con un sermón aburrido (Solís, 2011). Se aconseja también emplear en la hechura del archivo dos o más voces: intercalar timbres masculinos y femeninos, de preferencia (Marcelo y Martín, 2008).

Al respecto, hay que decir que un error recurrente en radio convencional, que se traslada a la producción de podcasts -con el agravante de que en la escucha de éstos se hace aún más evidente- es el de querer el entrevistador ser más protagónico que el entrevistado. Algo que demeritará sin duda el producto final. Por otro lado, hay que evitar engolar demasiado la voz: una entonación natural será más agradable y mejor apreciada por el escucha. Teniendo siempre en mente que se deben hacer cambios de entonación, a fin de no tener un ritmo y timbre demasiado monótono.

Finalmente, se debe buscar que el podcast sea distintivo de manera positiva: tocar temáticas originales, abrir el audio con una identificación -tanto de la emisión, como de los participantes-, intercalar espacios con música, e incluir efectos, o sonido ambiental (McComas, 2005).

\subsubsection{Cómo se difunde un podcast}

La Web 2.0 implica un reto para cualquier organización que emita noticias. El éxito de la prensa digital dependerá de la capacidad de las agencias de crear sitios que permitan la interacción de usuarios con los contenidos, y entre los propios usuarios. También se espera que los comunicadores sociales den el uso adecuado a las herramientas Web 2.0 para acercar la noticia a los usuarios potenciales (RodríguezMartínez y Pedraza-Jiménez, 2009). De ahí la importancia de difundir los contenidos informativos a través de espacios virtuales como las redes sociales.

Para la difusión de información, las redes aprovechan, administran y optimizan la utilización del espacio digital y la conectividad entre los usuarios. De esta manera, hay un rendimiento mayor en las conexiones y transferencias; se comparten recursos generados por algún servicio o aplicación (Caldevilla, Del Valle, \& Del Valle, 2012). Frecuentemente, se utilizan para compartir ficheros de cualquier tipo, como los audios, y en este caso concreto, los podcasts.

Según Nicolás (2015) la comunidad hispanoparlante es una de las más signficativas en el mundo en cuanto al consumo de podcast. Los países que escuchan y/o 
descargan más este tipo de recursos son: España, México, Colombia, Argentina y Chile. Asimismo, señala que, al ser el podcast un medio de consumo no inmediato, su impacto real se puede prolongar hasta por cuatro semanas. Ahora bien, un audio que alcance más de 180 descargas o reproducciones se considera bien colocado; y cuando alcanza las 1200, está por encima de la media. Por estas circunstancias, cada vez más profesionistas están prefiriendo utilizar estas herramientas para la difusión de información de diversa índole a sus seguidores, al ser una forma muy eficiente de comunicar por un bajo costo mensajes a su audiencia meta. Estos resultados permiten ser optimistas respecto al potencial de difusión de los contenidos expresados a través de un podcast, en cuanto al impacto deseado.

Otro aspecto que denota el potencial de esta herramienta digital es el hecho de que el encontrar un podcast determinado -o de una temática deseada- no presenta dificultad mayor, al existir diversos buscadores especializados -en una gran cantidad de idiomas- para este tipo de archivos, divididos en categorías específicas. Además, muchos de los podcasts tienen la posibilidad de ser descargados de manera gratuita (Ramos y Caurcel, 2011).

Por otra parte, los archivos de audio publicados se pueden asociar a Sindicación Realmente Simple (RSS, por sus siglas en inglés) o agregador web. De este modo, el usuario queda suscrito a una página web determinada -periódico, blog, wiki, entre otros- quien recibirá una notificación cada vez que se publique algo nuevo, o se actualice la información (Johnson y Grayden, 2006).

Para obtener un óptimo nivel de respuesta y audiencia, es conveniente establecer un período específico de publicación del podcast-diario, semanal, quincenal-; y respetar la periodicidad establecida, a fin de que el usuario tenga la seguridad de encontrar contenido nuevo cada vez que lo espera (Tenorio, 2008).

Por último, hay que decir que el podcast es realmente útil en la difusión de pláticas, ponencias, cátedras y entrevistas de profesionales y expertos, resultado de su gran facilidad para resguardarse en cualquier sitio web, así como compartirse desde el mismo (Laaser, Jaskilioff, Rodríguez y Lía, 2010).

\section{OBJETIVOS}

Los objetivos que se plantearon fueron:

Objetivo general:

Evaluar los podcasts de noticias que se publican en plataformas digitales de emisoras de radio de la ciudad de Puebla.

Objetivos específicos:

a. Identificar los elementos técnicos que se implementan en producción del podcast.

b. Delimitar los aspectos de contenido (función) que se incluyen en los podcasts publicados. 
Rivera Salas, P. E., y Montoya Márquez, O. R. Evaluación de los podcasts de noticias: un acercamiento a emisoras de la ciudad de Puebla

c. Establecer los mecanismos de difusión que se utilizan para dar a conocer las grabaciones.

\section{METODOLOGÍA}

La investigación se realizó bajo un enfoque cualitativo, puesto que este documento se construyó a partir de las experiencias que reportan los distintos podcasts que se consideraron en la muestra (Sáenz-López \& Rodríguez-Burgos, 2014). Además, es de tipo exploratoria dado que es una temática poco abordada. No se realizó ninguna manipulación de variables, por lo que es una investigación no experimental. Como la información se recabó en un sólo momento, se considera como de cohorte transversal, puesto que los podcasts que se incluyeron son las grabaciones que se han publicado hasta el segundo semestre de 2017 (Hernández Sampieri, Fernández Collado \& Baptista, 2014).

Para la recolección de datos, se utilizó la observación directa. Esta técnica se implementó ya que representa un hecho cotidiano que forma parte de la percepción de los acontecimientos en el día a día (Díaz, 2011). Además, se recurrió a la fuente primaria para la recolección de datos, en este caso, las grabaciones publicadas (Centeno-Maldonado \& de la Garza - Montemayor, 2014).

Adicionalmente, la construcción de la guía de observación se realizó a partir del análisis de diversos documentos que se recabaron mediante bases de datos de artículos científicos -Scielo, Dialnet, Redalyc, DOAJ-. La búsqueda se ejecutó considerando como términos clave las palabras: Podcast, Podcasting, Periodismo digital y Ciberperidismo. Esta búsqueda permitió delimitar el instrumento que sustenta esta investigación. Además, se implementaron las siguientes categorías para la evaluación de las grabaciones: producción, función periodística y difusión.

La muestra se integró por tres radiodifusoras privadas y una pública, todas localizadas en la ciudad de Puebla, México. Estas organizaciones fueron consideradas en virtud de que afirman en sus sitios electrónicos y en su publicidad contar con podcasts que difunden las noticias que se transmiten en sus programas. Cabe señalar que, aunque otras dos radiodifusoras particulares reportaron tener podcast, no fueron incluidas al no tener sus links habilitados para su consulta. Los resultados se presentan en los siguientes apartados.

\section{DISCUSIÓN}

\subsection{Análisis de la producción de los podcasts}

Como se mencionó anteriormente, la producción de un podcast considera aspectos medulares para que la grabación sea atractiva y tenga un buen impacto. Para este grupo observado, se aprecia que los podcasts tienden a ser largos, con una duración promedio de 140 minutos. Sólo una de las emisoras publica podcasts que se extienden hasta un máximo de 5 minutos. Por otro lado, las emisoras no registran el número de grabaciones que tienen de su programa de noticias: sólo una de ellas señaló haber acumulado 1,313 grabaciones hasta el mes de diciembre de 2017. 
En cuanto a la calidad del sonido, la emisora estatal y la Radiodifusora 1 tuvieron un alto nivel, mientras que las dos emisoras restantes tuvieron una calidad media: se perciben en estas últimas mucho eco y ruido a lo largo de la emisión. Además, la grabación no se incluye completa o se integra parte de la grabación del siguiente programa. Estos elementos pueden conllevar a que el receptor deje de interesarse por el programa o lo escuche parcialmente, tal y como lo señala McComas (2005).

Evaluando las características que las grabaciones tienen, todas las emisoras cumplen con delimitar su público meta, tener un sello distintivo y un correcto balance entre la voz en off y los demás sonidos que visten el podcast. En términos de esta categoría, la emisora que genera un podcast con mejor producción es la emisora del estado. La característica que no se cumple para la mayoría de las unidades observadas es la presentación de la información. Esto es porque los podcasts que se tienen almacenados son simples grabaciones de la emisión en vivo, como lo estipula Solís, (2010). Por lo tanto, no se realiza un resumen de la emisión, de forma que el receptor se interese por escuchar la emisión completa. La tabla 1 sintetiza el seguimiento que se hizo al respecto de la producción de los podcasts.

Tabla 1. Producción de un podcast.

\begin{tabular}{|c|c|c|c|c|}
\hline \multirow[b]{2}{*}{ Características observadas } & \multicolumn{3}{|c|}{$\begin{array}{l}\text { Emisoras } \\
\text { privadas }\end{array}$} & \multirow[b]{2}{*}{$\begin{array}{l}\text { Emisora } \\
\text { pública }\end{array}$} \\
\hline & 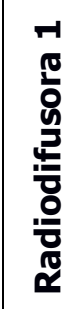 & 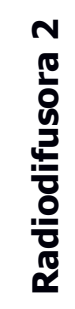 & 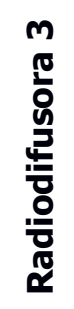 & \\
\hline Tiene un público meta -audiencia como colectivo y como individuo-. & Si & $\mathrm{Si}$ & $\mathrm{Si}$ & $\mathrm{Si}$ \\
\hline Se identifica la fecha de producción del podcast. & $\mathrm{Si}$ & No & No & $\mathrm{Si}$ \\
\hline $\begin{array}{l}\text { Plantea la información de manera creativa, recordando que es otra } \\
\text { forma de llevar la información al público. }\end{array}$ & $\mathrm{Si}$ & No & No & No \\
\hline $\begin{array}{l}\text { Existe un formato para promover el programa o emisión, y/o la } \\
\text { estación de radio. }\end{array}$ & $\mathrm{Si}$ & $\mathrm{Si}$ & No & $\mathrm{Si}$ \\
\hline $\begin{array}{l}\text { Inserta elementos auditivos que enfaticen la información clave -pueden } \\
\text { ser grabaciones de entrevistados, de los hechos que se están } \\
\text { narrando, entre otros-. }\end{array}$ & No & $\mathrm{Si}$ & $\mathrm{Si}$ & $\mathrm{Si}$ \\
\hline $\begin{array}{l}\text { Cuenta con un sello distintivo congruente con la emisora, el periodista } \\
\text { y/o el reportero. }\end{array}$ & $\mathrm{Si}$ & $\mathrm{Si}$ & $\mathrm{Si}$ & $\mathrm{Si}$ \\
\hline Se inserta publicidad en el podcast. & No & $\mathrm{Si}$ & $\mathrm{Si}$ & $\mathrm{Si}$ \\
\hline Incluye ideas cortas y concretas. & $\mathrm{Si}$ & $\mathrm{Si}$ & $\mathrm{Si}$ & $\mathrm{Si}$ \\
\hline $\begin{array}{l}\text { Se aprecian "sonidos naturales" o música de fondo que ayudan a } \\
\text { enganchar a la audiencia. }\end{array}$ & $\mathrm{Si}$ & No & No & $\mathrm{Si}$ \\
\hline Hay un correcto balance entre la música y efectos, y la voz en off. & $\mathrm{Si}$ & $\mathrm{Si}$ & $\mathrm{Si}$ & $\mathrm{Si}$ \\
\hline $\begin{array}{l}\text { Integra música de fondo para ayudar a fomentar la identidad tanto con } \\
\text { la emisora como con el portal de noticias. }\end{array}$ & No & No & $\mathrm{Si}$ & $\mathrm{Si}$ \\
\hline
\end{tabular}

Fuente: Elaboración propia con datos de la muestra. 


\subsection{La función periodística del podcast}

Esta categoría reporta básicamente la función que realiza el podcast. Todas las emisoras analizadas difunden podcasts que introducen a la audiencia a la siguiente emisión, resumen las noticias y tienen un enfoque informativo. En este sentido, se percibe que lo postulado por Laaser, Jaskilioff, Rodríguez y Lía (2010) es aplicado por estos productores de contenidos. La Radiodifusora 1 no profundiza en algún tema en específico, en comparación con las otras tres emisoras que se analizaron. Destaca en esta categoría que el enfoque descriptivo y de opinión no son retomados. La tabla 2 resume estas apreciaciones.

Tabla 2. Función del podcast.

\begin{tabular}{|c|c|c|c|c|}
\hline \multirow[b]{2}{*}{ Características observadas } & \multicolumn{3}{|c|}{$\begin{array}{l}\text { Emisoras } \\
\text { privadas }\end{array}$} & \multirow[b]{2}{*}{$\begin{array}{l}\text { Emisora } \\
\text { pública }\end{array}$} \\
\hline & 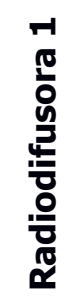 & 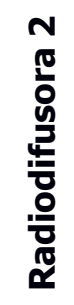 & 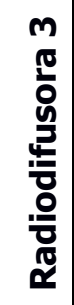 & \\
\hline Introduce a la audiencia para la siguiente emisión. & Si & $\mathrm{Si}$ & $\mathrm{Si}$ & Si \\
\hline Resume las noticias actuales. & $\mathrm{Si}$ & $\mathrm{Si}$ & $\mathrm{Si}$ & $\mathrm{Si}$ \\
\hline Profundiza en un tema en específico. & No & $\mathrm{Si}$ & $\mathrm{Si}$ & $\mathrm{Si}$ \\
\hline Tiene un enfoque informativo. & $\mathrm{Si}$ & $\mathrm{Si}$ & $\mathrm{Si}$ & $\mathrm{Si}$ \\
\hline Tiene un enfoque descriptivo. & No & No & No & No \\
\hline Tiene un enfoque de opinión. & No & No & No & No \\
\hline
\end{tabular}

Fuente: Elaboración propia con datos de la muestra.

Complementario a lo anterior, se identificaron los géneros periodísticos que se incluyen en las grabaciones de manera cotidiana -revise tabla 3-. Sobresale como género más socorrido la nota informativa, seguido del comentario y el reportaje. Es precisamente este último uno de los señalados como preferidos para posicionarse dentro del podcast (Izquierdo-Labella, 2012; Briggs, 2007).

Tabla 3. Géneros contenidos en los podcasts.

\begin{tabular}{|c|c|c|c|c|}
\hline \multirow[b]{2}{*}{ Géneros } & \multicolumn{3}{|c|}{ Emisoras privadas } & \multirow[b]{2}{*}{$\begin{array}{l}\text { Emisora } \\
\text { pública }\end{array}$} \\
\hline & 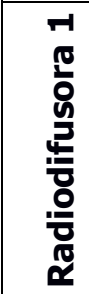 & 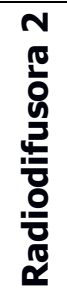 & 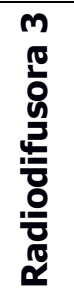 & \\
\hline Reportaje & & & & \\
\hline
\end{tabular}




\begin{tabular}{|c|l|l|l|l|}
\hline Crónica & & & & \\
\hline Entrevista & & & & \\
\hline Nota informativa & & & & \\
\hline Artículo & & & & \\
\hline Editorial & & & & \\
\hline Columna & & & & \\
\hline Comentario & & & & \\
\hline
\end{tabular}

Fuente: Elaboración propia con datos de la muestra.

\subsection{Difusión del podcast}

Esta categoría es muy significativa, pues de aquí se parte para dar a conocer de forma adecuada los productos que se generan. Para esta categoría llama la atención que la única característica que cumplen todas las pistas revisadas es "Ocupan otros sitios en la Web relacionados con la empresa para promover el podcast". Por otro lado, las coincidencias se detectan en elementos que no cubren las unidades de observación. Así, ninguna de las emisoras utiliza títulos llamativos, ni un resumen. Tampoco se distingue interacción en los foros que acompañan a los podcasts, ni el uso del código RSS como lo recomiendan ciertos autores (Johnson y Grayden, 2006). El podcast publicado por la Radiodifusora 1 es el que tiene mejor difusión de acuerdo con los parámetros planteados en la investigación -para mayor precisión, revisar la tabla 4-.

Tabla 4. Características reportadas de la difusión de los podcasts.

\begin{tabular}{|c|c|c|c|c|}
\hline \multirow[b]{2}{*}{ Características observadas } & \multicolumn{3}{|c|}{ Emisoras privadas } & \multirow[b]{2}{*}{$\begin{array}{l}\text { Emisora } \\
\text { pública }\end{array}$} \\
\hline & 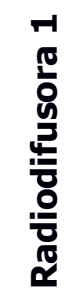 & 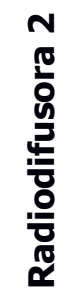 & 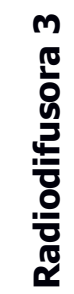 & \\
\hline Las ligas (links) del podcast están disponibles. & $\mathrm{Si}$ & $\mathrm{Si}$ & No & $\mathrm{Si}$ \\
\hline $\begin{array}{l}\text { Se agregan etiquetas para el correcto posicionamiento del } \\
\text { podcast. }\end{array}$ & $\mathrm{Si}$ & No & No & No \\
\hline Se describe el podcast brevemente. & No & $\mathrm{Si}$ & No & No \\
\hline $\begin{array}{l}\text { Se aprecia un título llamativo y un resumen para atraer a la } \\
\text { audiencia. }\end{array}$ & No & No & No & No \\
\hline $\begin{array}{l}\text { Agregan una imagen alusiva al contenido que será } \\
\text { escuchado. }\end{array}$ & No & No & No & $\mathrm{Si}$ \\
\hline $\begin{array}{l}\text { Publican el enlace al podcast y/o al portal de noticias en redes } \\
\text { sociales. }\end{array}$ & $\mathrm{Si}$ & $\mathrm{Si}$ & No & No \\
\hline $\begin{array}{l}\text { Promueven el podcast durante la emisión, o dentro de la } \\
\text { programación. }\end{array}$ & $\mathrm{Si}$ & No & No & No \\
\hline Establecen un foro de discusión sobre el podcast. & $\mathrm{Si}$ & No & No & $\mathrm{Si}$ \\
\hline Hay interacción en los foros de discusión sobre el podcast. & No & No & No & No \\
\hline Insertan código RSS para que la audiencia le dé seguimiento. & No & No & No & No \\
\hline
\end{tabular}


Rivera Salas, P. E., y Montoya Márquez, O. R. Evaluación de los podcasts de noticias: un acercamiento a emisoras de la ciudad de Puebla

\begin{tabular}{|l|c|c|c|c|}
\hline $\begin{array}{l}\text { Ocupan otros sitios en la Web relacionados con la empresa } \\
\text { para promover el podcast. }\end{array}$ & $\mathrm{Si}$ & $\mathrm{Si}$ & $\mathrm{Si}$ & $\mathrm{Si}$ \\
\hline Existe la posibilidad de consultar podcasts anteriores. & $\mathrm{Si}$ & $\mathrm{No}$ & $\mathrm{No}$ & $\mathrm{Si}$ \\
\hline
\end{tabular}

Fuente: Elaboración propia con datos de la muestra.

A la par, se revisaron las redes sociales con las que los podcasts están vinculados. En la tabla 5 se resalta que Facebook es el espacio virtual al que más se recurre. El segundo lugar lo ocupa Twitter. Por otra parte, el podcast de la Radiodifusora 1 se vincula con cuatro redes sociales, incluyendo Pinterest, un sitio especializado en imágenes. Lo cual llama particularmente la atención.

Tabla 5. Redes sociales utilizadas para la difusión de los podcasts.

\begin{tabular}{|c|c|c|c|c|}
\hline \multirow[b]{2}{*}{ Red social } & \multicolumn{3}{|c|}{$\begin{array}{l}\text { Emisoras } \\
\text { privadas }\end{array}$} & \multirow[b]{2}{*}{$\begin{array}{l}\text { Emisora } \\
\text { pública }\end{array}$} \\
\hline & 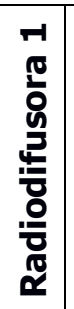 & 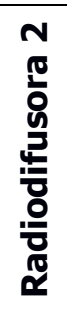 & 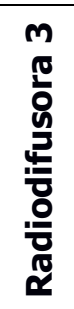 & \\
\hline \multicolumn{5}{|l|}{ Twitter } \\
\hline \multicolumn{5}{|l|}{ Facebook } \\
\hline \multicolumn{5}{|l|}{ Pinterest } \\
\hline Google + & & & & \\
\hline
\end{tabular}

Fuente: Elaboración propia con datos de la muestra.

\section{CONCLUSIONES}

A partir de la revisión realizada se infiere que el podcast es una herramienta digital sumamente versátil (Quintanal, 2012), que se ha posicionado paulatinamente como un recurso que las radiodifusoras cada vez más consideran importante publicar.

En cuanto a la producción del podcast, se ha realizado comúnmente como poco más que una simple copia de las emisiones de los noticieros de la radio tradicional. No obstante, a pesar de que no es aconsejable una grabación de tan larga duración, es consultada por su audiencia de manera regular y prometedora. Además, al integrar todos los contenidos -incluyendo la publicidad- se genera un mecanismo eficaz para colocar productos y servicios.

Se observa que la principal función del podcast -al menos en el caso de las radiodifusoras que se analizaron en el estudio- es de un eminente enfoque informativo, e incluye a unos pocos géneros (Salaverría, 2005). Por lo que es importante que los productores evalúen la posibilidad de insertar otros enfoques que motiven la interacción con los usuarios, mediante la generación de polémica -como el 
Rivera Salas, P. E., y Montoya Márquez, O. R. Evaluación de los podcasts de noticias: un acercamiento a emisoras de la ciudad de Puebla

artículo de investigación, la editorial, o el artículo de opinión-, pero que al mismo tiempo inviten a la reflexión y a la crítica ciudadana. Con ello se contribuiría a cumplir con las funciones del periodismo dentro de la sociedad mediatizada de hoy.

Adicionalmente, la difusión del podcast debe llevarse a cabo de manera más estratégica. Por un lado, se nota la falta de utilización del código RSS, el cual notifica al usuario sobre las nuevas emisiones y actualizaciones que se han insertado en el espacio digital de determinado sitio web de su interés. Hacer llegar actualizaciones pertinentes a los consumidores facilita su fidelización hacia la página web, el blog, la emisión, o el podcast mismo (Johnson y Grayden, 2006).

Al mismo tiempo, es indispensable que se describa someramente el contenido del podcast (Laaser, Jaskilioff, Rodríguez y Lía, 2010), así como identificarlo mediante una imagen relacionada a la temática, para lograr enganchar a mayor audiencia. Por otro lado, el amplio espectro de redes sociales existentes en el ciberespacio debe ser utilizado para colocar los podcasts. Si bien la red social Facebook constituye un soporte de gran utilidad, existen otras opciones que permitirían a las radiodifusoras llegar a un número mayor de ciberescuchas.

Finalmente, hay que decir que, aunque es notorio el avance que el podcast ha obtenido en los últimos años, llama la atención que las grandes emisoras de la ciudad de Puebla (México), aún no adoptan de manera sistemática esta herramienta como parte de los recursos que ofrecen a su público. Es importante que se valore el potencial de este instrumento para poder alcanzar una mayor cuota de mercado, sobre todo en una era en la que el formato digital está irrumpiendo en los espacios que los medios tradicionales habían acaparado.

\section{REFERENCIAS}

Briggs, M. (2007). Periodismo 2.0. Maryland, Estados Unidos de América: John S. and James L. Knight Foundation.

Bullido, E. (9 de Marzo de 2015). El renacimiento de los podcasts, una oportunidad para el periodismo. Recuperado de La pirámide invertida: http://enriquebullido.com/2015/03/09/el-renacimiento-de-los-podcastsoportunidad-para-el-periodismo/

Caldevilla, D., Del Valle, M. E., \& Del Valle, C. (2012). Las radios 2.0: Redes sociales que precisan de un marco ético de regulación. La ética de la comunicación a comienzos del siglo XXI: I Congreso Internacional de Ética de la Comunicación (pp. 1239-1252). Sevilla, España: Edufora.

Centeno-Maldonado, J. C., \& de la Garza - Montemayor, D. J. (2014). Observación. En K. Sáez - López , \& G. Taméz-González, Metodos y técnicas cualitativas y cuantitativas aplicables en la investigación en Ciencias Sociales (pp. 202-221). Ciudad de México: Tirant Humanidades México. 
Rivera Salas, P. E., y Montoya Márquez, O. R. Evaluación de los podcasts de noticias: un acercamiento a emisoras de la ciudad de Puebla

Díaz, L. (2011). La observación. Ciudad de México: Facultad de Psiciología (Textos de apoyo didáctico) UNAM.

Díaz-Noci, J., \& Salaverría, R. (2003). Hipertexto periodístico: Teoría y modelos. En J. Díaz-Noci, \& R. Salaverría, Manual de redacción ciberperiodística (pp. 82-139). Barcelona, España: Ariel.

Hernández Sampieri, R., Fernández Collado, C., \& Baptista, M. P. (2014). Metodología de la investigación. Ciudad de México: Mc Graw Hill.

Izquierdo-Labella, L. (2012). Comunicación Glocal. El periodismo local abre una ventana al mundo. Valencia, España: Tirant Lo Blanch.

Jarque-Muñoz, J. M., \& Almiron-Roig, N. (2008). Periodismo para Internet o periodismo, y punto. Palabra clave, 11(2), 219-252.

Johnson, L., \& y Grayden, S. (2006). Podcasts: an emerging form of digital publishing. Internacional Journal of Computerized Dentristy, 9(3), 205-218.

Laaser, W., Jaskilioff, S. L., Rodríguez, B., \& y Lía, C. (2010). Podcasting: ¿Un nuevo medio para la Educación a Distancia? RED. Revista de Educación a Distancia, 23, $1-11$.

Marcelo, J., \& Martín, E. (2008). Podcasting. Guía práctica. Madrid, España: Anaya Multimedia.

McComas, C. (2009). 20 Ideas for an Excellent Podcast. Recuperado de http://www.workhappy.net/2009/02/20-ideas-for-an-excellent-podcast.html

Nicolás, C. (2015). 062 Analizando las estadísticas de un podcast con Elsie Escobar. Recuperado de Sueldo 3.0: http://www.sueldo30.com/estadisticas-podcast-conelsie-escobar/

Quintanal, F. (2012). El podcast como herramienta de enseñanza en física y química de bachillerato. Estudios sobre el Mensaje Periodístico, 18, 729-738.

Ramos, A. M., \& Caurcel, M. D. (2011). Los podcast como herramientas de enseñanza-apredizaje en la universidad. Revista de Curriculum y Formación de Profesorado, 151-162.

Rodríguez-Martínez, R., \& Pedraza-Jiménez, R. (2009). Prensa digital y Web 2.0. Recuperado de Hipertext.net: https://www.upf.edu/hipertextnet/numero7/prensa-digital.html

Sáenz-López, K. A., \& Rodríguez-Burgos, K. E. (2014). Habilidades Investigativas. En K. Sáenz-López, \& G. Taméz-González, Métodos y técnicas cualitativas y cuantitativas aplicables a las Ciencias Sociales (págs. 85-96). Ciudad de México: Tirant Humanidades México. 
Rivera Salas, P. E., y Montoya Márquez, O. R. Evaluación de los podcasts de noticias: un acercamiento a emisoras de la ciudad de Puebla

Salaverría, R. (2005). Redacción periodística en internet. Pamplona, España: Ediciones Universidad de Navarra.

Solano, I. M., \& Sánchez, M. M. (2010). Aprendiendo en cualquier lugar: El podcast educativo. Pixel-Bit, 125-139.

Solís, D. (2011). Podcasting fácil para docentes y alumnos. Un recurso más para el dominio de las Tic's en la educación. Ciudad de México: Grupo Cultural Argón.

Tenorio, I. (2008). Podcast: Manual de podcaster. Madrid, España: Marcomobo Ediciones Técnicas.

\section{AUTORES:}

\section{Paola Eunice Rivera Salas}

Profesora - Investigadora de la Facultad de Ciencias de la Comunicación de la Benemérita Universidad Autónoma de Puebla (BUAP); institución en la que también es parte del grupo de facilitadores de la Escuela de Formación Docente. Doctora en Educación (Universidad Virtual Hispánica de México). Maestra en Educación Superior Grado Cum Laude (BUAP). Especialista en Periodismo y Ciencias de la Información (Universidad Europea del Atlántico), y Licenciada en Economía (BUAP). Es docente perfil PRODEP Y CERTIDEMS. Miembro del Cuerpo Académico "Comunicación y Sociedad". Miembro de la Asociación Iberoamericana de Sociología de las Organizaciones y Comunicación (AISOC). Líneas de investigación: Educación, Comunicación y TIC. Conductora de radio por internet.

paola.rivera@correo.buap.mx

Orcid ID: https://orcid.org/0000-0002-8050-3585

\section{Octavio Ricardo Montoya Márquez}

Profesor universitario en el área de la comunicación y las tecnologías digitales. Maestrante en Comunicación Estratégica y Licenciado en Comunicación (Benemérita Universidad Autónoma de Puebla). Ha presentado diversas ponencias y ha publicado capítulos de libro dentro de la temática de las TIC y la Educación. Licenciado en Administración de Empresas (Instituto Tecnológico de Zacatecas). Su tesis de maestría es una investigación centrada en la interacción digital en la radio online; área en la que cuenta con amplia experiencia, siendo conductor de un programa radiofónico vía web desde hace más de tres años.

octaviomontoya95@gmail.com 